\title{
Multimedia Material to Help Photosynthesis Teaching in Regular and Distance Classes
}

\author{
Costa, G.R.T.; Milhomem, D.A.; Lima, C.M.R.; Castro, M.S. and Fontes, W.
}

Laboratório de Bioquímica e Química de Proteínas, Departamento de Biologia Celular, Universidade de Brasília, Brasília/DF wagnerf@unb.br

The difficulties related to the understanding of biochemistry concepts and processes observed during regular classes using only schemes and static pictures have raised the interest of professors towards multimedia material. In the few last decades we could notice the development of many computational tools that are helping this process. This work presents a software elaborated to show the phases of Photosynthesis (both light-dependent and independent processes). FLASH software was used to make regular classes more attractive with schemes, interactivity and animations to be projected. An intuitive navigation system was elaborated using ActionScript programming, so that students can use the same class material for reviewing the major topics. The students will benefit from characteristics like text boxes, summarizing the concepts displayed on each screen, the guided study mode that suggests to the student the next navigation step and the automatic navigation that gives a general look about the theme, yet keeping the freedom for choosing any available topic.

Support: FUB/UnB; FINATEC 